\title{
Genome-wide association mapping reveals novel sources of resistance to northern corn leaf blight in maize
}

\author{
Junqiang Ding ${ }^{1+}$, Farhan Ali ${ }^{1+}$, Gengshen Chen ${ }^{1}$, Huihui Li ${ }^{2}$, George Mahuku ${ }^{3}$, Ning Yang ${ }^{1}$, Luis Narro ${ }^{3}$, \\ Cosmos Magorokosho ${ }^{3}$, Dan Makumbi ${ }^{3}$ and Jianbing Yan $^{1 *}$
}

\begin{abstract}
Background: Northern corn leaf blight (NCLB) caused by Exserohilum turcicum is a destructive disease in maize. Using host resistance to minimize the detrimental effects of NCLB on maize productivity is the most cost-effective and appealing disease management strategy. However, this requires the identification and use of stable resistance genes that are effective across different environments.

Results: We evaluated a diverse maize population comprised of 999 inbred lines across different environments for resistance to NCLB. To identify genomic regions associated with NCLB resistance in maize, a genome-wide association analysis was conducted using 56,110 single-nucleotide polymorphism markers. Single-marker and haplotype-based associations, as well as Anderson-Darling tests, identified alleles significantly associated with NCLB resistance. The single-marker and haplotype-based association mappings identified twelve and ten loci (genes), respectively, that were significantly associated with resistance to NCLB. Additionally, by dividing the population into three subgroups and performing Anderson-Darling tests, eighty one genes were detected, and twelve of them were related to plant defense. Identical defense genes were identified using the three analyses.

Conclusion: An association panel including 999 diverse lines was evaluated for resistance to NCLB in multiple environments, and a large number of resistant lines were identified and can be used as reliable resistance resource in maize breeding program. Genome-wide association study reveals that NCLB resistance is a complex trait which is under the control of many minor genes with relatively low effects. Pyramiding these genes in the same background is likely to result in stable resistance to NCLB.
\end{abstract}

\section{Background}

Maize (Zea mays L.) is an important crop for food, feed and industry. Moreover, it is a model genetic system with many advantages, including its great levels of phenotypic and genetic diversity [1]. Identifying the natural allelic variations that lead to this phenotypic diversity will contribute to the improvement of agronomic traits in maize breeding. However, dissecting quantitative traits poses numerous challenges that make gene identification more difficult, including the limitations of molecular biology and bioinformatics tools [2]. Rapid developments in genome-wide

\footnotetext{
*Correspondence: yjianbing@mail.hzau.edu.cn

${ }^{\dagger}$ Equal contributors

${ }^{1}$ National Key Laboratory of Crop Genetic Improvement, Huazhong

Agricultural University, Wuhan 430070, China

Full list of author information is available at the end of the article
}

association mapping, combined with an extensive array of genome resources and technologies, have increased the power and accuracy to dissect complex traits and identify alleles associated with quantitative trait loci (QTL) for important agronomic traits [1, 3]. Recently, association mapping has become an influential approach for dissecting complex traits of interest. Distinct from the genetic analyses in segregating populations, genome-wide association study (GWAS) is based on the accurate phenotyping of a particular trait in a huge set of individuals that are widely unrelated (i.e., they have little or no family structure). For this reason, association mapping has been extensively used to study the genetic bases of complex traits in plant and animal systems $[1,4,5]$.

Dissecting the genetic bases of different traits is the foundation of trait improvement; however, despite the recent 
advancements in this area, very little is known about the genetic architecture of many adaptive traits in maize [6], especially resistance to northern corn leaf blight (NCLB) and several other diseases. NCLB is caused by a hemibiotrophic fungal pathogen, Exserohilum turcicum (teleomorph Setosphaeria turcica) [7]. This disease is prevalent in maize growing areas worldwide and is associated with moderateto-severe yield losses [8]. A severe NCLB infection prior to flowering may cause $>50 \%$ losses in maize final yields [9]. The most economical and effective strategy for managing NCLB is the use of genetic resistance. The genetics of NCLB resistance have been extensively studied using biparental populations but are still poorly understood because of several factors, including low marker densities and the small population sizes used in many studies. A QTL analysis typically produces a large confidence interval, and it is usually uncertain whether a QTL corresponds to one or multiple linked genes $[10,11]$. Until recently, only a small number of causal genes underlying large-effect QTLs have been identified and cloned in cereals [6].

In view of the potential power of association mapping to dissect the genetics of complex traits, and the problems of QTL mapping, this study was undertaken to shed light on the genetic architecture of NCLB resistance and to identify resistance-associated genes in globally collected diverse maize germplasm.

\section{Results}

\section{Phenotypic diversity}

A global collection of 999 diverse inbred lines from the International Maize and Wheat Improvement Center (CIMMYT) germplasm collection was used for association mapping (Additional file 1: Table S1). Three related NCLB traits, mean rating, high rating and the area under the disease progress curve (AUDPC), were adopted to comprehensively evaluate the resistance to NCLB in association panel in 12 environments (Additional file 2: Table S2). The analysis of variance for NCLB resistance revealed significant differences $(\mathrm{P} \leq 0.01)$ and high heritabilities for all of the traits under investigation (Table 1). Correlation results showed high positive associations between these traits. A maximum correlation value of 0.99 was observed between the mean rating and AUDPC, whereas the lowest value $(r=0.93)$ was observed between the high rating and AUDPC. No line was observed to be completely resistant to this disease, and most of the lines fell into the middle category (Fig. 1). The five highly resistant inbred lines were CIMBL225, CML305, CIMBL399, CML483 and CIMBL269, whereas the most susceptible lines were CML130, CML112 and CIMBL43 (Additional file 1: Table S1). These lines can be used as controls in future NCLB phenotyping studies and as parents to develop biparental populations for molecular breeding and marker-assisted selection.

\section{Familial relatedness among lines}

The 56,110 markers used in this study were used in different analyses, including principal component analyses (PCA), structure (Q) and kinship (K) analyses, to determine the relationships among the individuals in this association panel. The first 10 principal components in this association panel were shown to control $14.7 \%$ of the cumulative variance, with each of them account for $0.7 \%-6.0 \%$ of the phenotypic variance (Additional file 3: Table S3). We also analyzed the data using STRUCTURE software to determine familial relatedness, and three subgroups were observed with $>50 \%$ possibility in each group (Additional file 4: Figure S1a). The K analysis also revealed that the 56,110 markers controlled $42.3 \%$, $47.4 \%$ and $53.8 \%$ of the total genetic variance for AUDPC, mean rating and high rating, respectively (Additional file 4 : Figure $\mathrm{S} 1 \mathrm{~b}, \mathrm{c}$ and $\mathrm{d}$ ).

\section{Genetic basis revealed by GWAS}

The SNP-based GWAS was performed using mixed linear model $(\mathrm{MLM})$ with rare alleles $(\mathrm{MAF}<5 \%)$ excluded, and both population structure (first 10 principle components) and kinship (K) were taken into account to avoid spurious associations. As is shown by the quantile-quantile plots (QQ plots) and Manhattan plots (Fig. 2), significant trait-marker associations that reached Bonferroni correction of $\mathrm{P} \leq 2.15 \times 10^{-5}(P<1 / \mathrm{n} ; n=$ total markers used) were observed. The number of significant markers revealed for AUDPC was 12, whereas 14 and 19 markers were associated with mean rating and high rating, respectively (Tables 2, 3 and 4). The number of significant loci varied from chromosome to chromosome, and each

Table 1 Analysis of variance, heritability and correlation

\begin{tabular}{|c|c|c|c|c|c|c|}
\hline \multirow[t]{2}{*}{ Traits } & \multicolumn{2}{|l|}{ Mean squares } & \multirow[t]{2}{*}{$\mathrm{H}^{2 \mathrm{~b}}$} & \multicolumn{3}{|l|}{ Correlation } \\
\hline & $\overline{\mathrm{E}^{\mathrm{a}}}$ & $G^{a}$ & & High rating & Mean rating & $\overline{A U D P C}$ \\
\hline High Rating & $116.98^{* *}$ & $2.35^{* *}$ & 0.83 & 1 & & \\
\hline Mean Rating & $112.44^{* *}$ & $1.10^{* *}$ & 0.76 & $0.94^{* *}$ & 1 & \\
\hline AUDPC & $424481.57^{* *}$ & $2012.67^{* *}$ & 0.76 & $0.93^{* *}$ & $0.99^{* *}$ & 1 \\
\hline
\end{tabular}

**Significant at $P \leq 0.01$

${ }^{a}$ Mean square values split into environmental and genotypic mean square ( $E$ and $G$ )

${ }^{\mathrm{b}}$ Stands for broad-sense heritability 

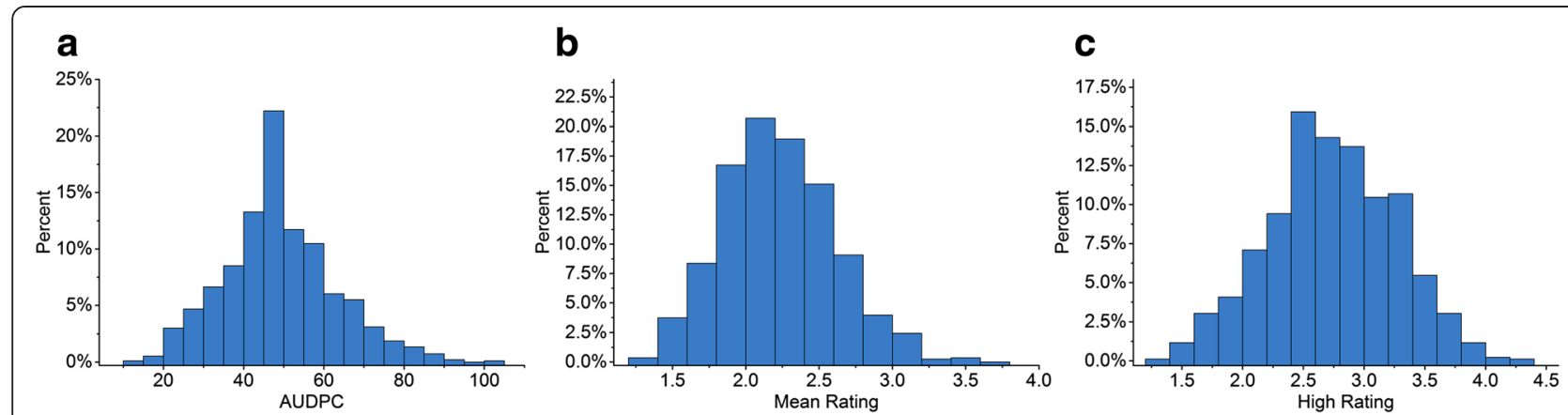

Fig. 1 Frequency distribution of phenotypic variation of resistance to NCLB. The frequency distributions of area under disease progress curve (AUDPC), Mean Rating and High Rating are shown in $\mathbf{a}, \mathbf{b}$ and, $\mathbf{c}$, respectively

locus explained a small portion (2\%-3\%) of phenotypic variation. The maximum candidate loci were observed on chromosome 7 for the AUDPC and mean rating, whereas chromosome 3 and 4 each had seven significant loci for high rating. Based on the physical locations of significant SNPs on the B73 reference genome sequence, the concerning candidate genes lying in the significant loci were identified, which included five, seven and seven genes conferring resistance for AUDPC, mean rating and high rating, respectively. In total twelve unique genes were detected for at least one resistance trait. Five identical genes associated with two or three resistance traits were observed as revealed by their strong phenotypic correlations, which included one gene on chromosome 4 (GRMZM2G171605), two genes on chromosome 7 (GRMZM2G100107 and GRMZM2G151651) and two genes on chromosome 10 (GRMZM2G158141 and GRM ZM2G020254). More importantly, functional annotations of the five genes showed that three of them related to plant defense. For example, GRMZM2G100107 was

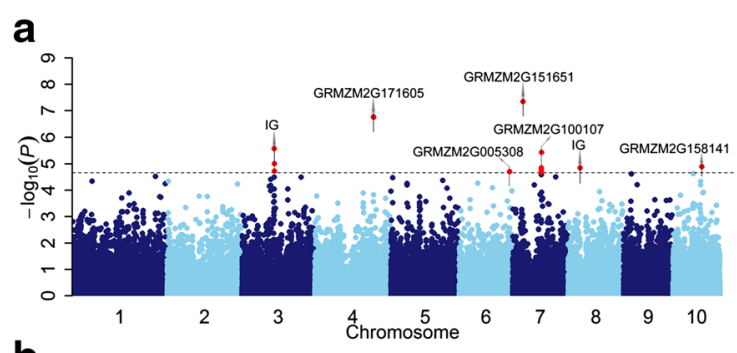

b。
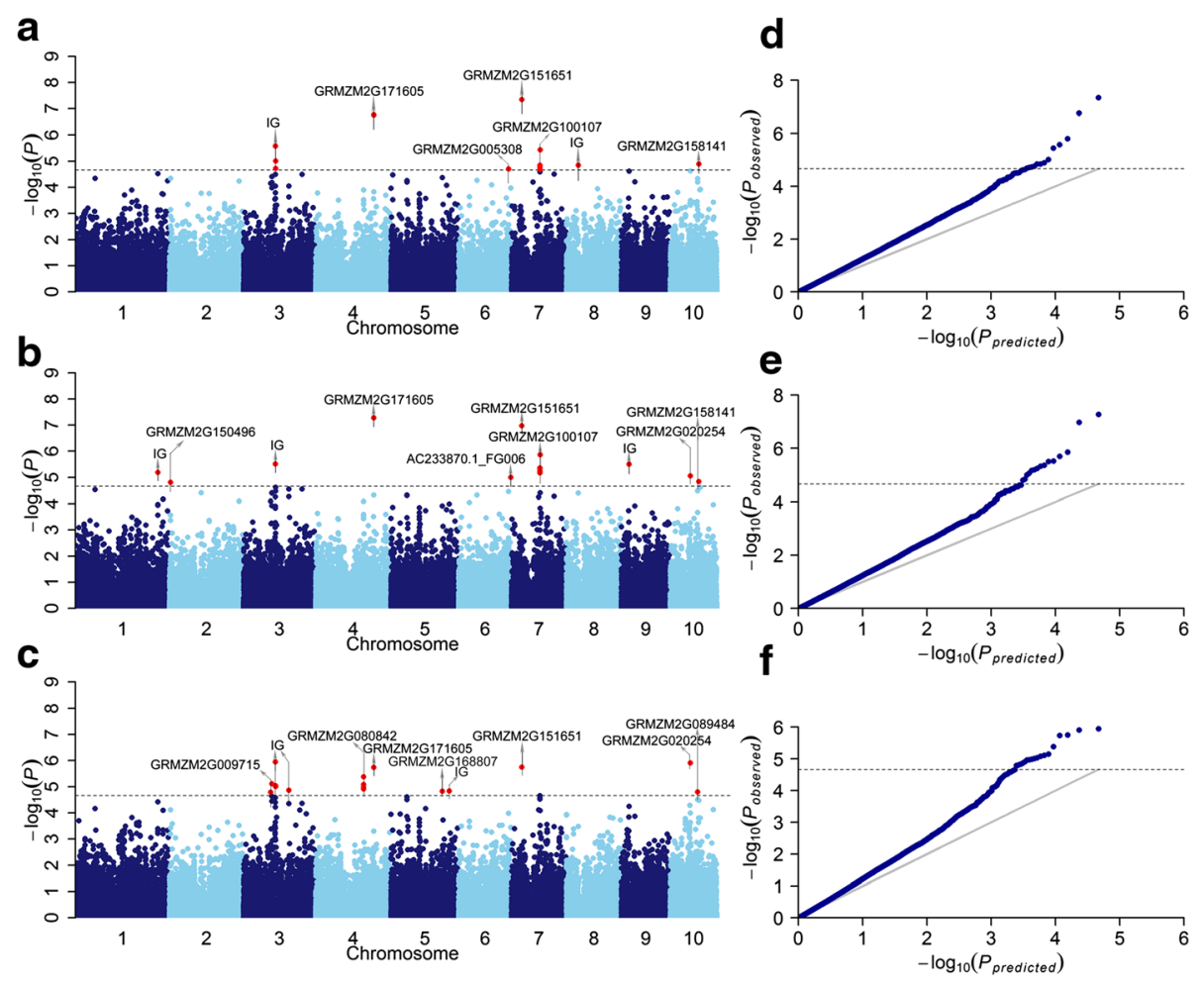

Fig. 2 Manhattan plots and QQ plots resulting from the SNP-based GWAS for AUDPC, Mean Rating and High Rating. Manhattan plots for area under disease progress curve (AUDPC), Mean Rating and High Rating are shown in $\mathbf{a}, \mathbf{b}$ and $\mathbf{c}$, respectively. QQ plots for area under disease progress curve (AUDPC), Mean Rating and High Rating are shown in $\mathbf{d}$, e and $\mathbf{f}$, respectively. The genes that reach Bonferroni correction of $\mathrm{P} \leq 2.15 \times 10^{-5}$ are listed, and IG stands for intergenic which means no gene is identified 
Table 2 Candidate genes, chromosomal position and SNPs significantly associated with Area under Disease Progress Curve (AUDPC) detected by SNP-based GWAS

\begin{tabular}{|c|c|c|c|c|c|c|c|c|c|c|}
\hline No. & Candidate gene & Chromosome & $\begin{array}{l}\text { Physical position } \\
\text { (AGP v.2) }\end{array}$ & SNP & Allele & $P$ value & $\mathrm{FDR}^{*}$ & $M F^{a}$ & $R^{2}$ & Annotation \\
\hline 1 & Intergenic & 3 & 103166745 & PZE-103062307 & $A, G$ & 2.67E-06 & 0.031 & 0.09 & 0.02 & \\
\hline 2 & Intergenic & 3 & 103544700 & PZE-103062210 & $A, G$ & $1.88 \mathrm{E}-05$ & 0.074 & 0.17 & 0.02 & \\
\hline 3 & Intergenic & 3 & 103769943 & PZE-103062159 & $A, C$ & 9.87E-06 & 0.074 & 0.13 & 0.02 & \\
\hline 4 & GRMZM2G171605 & 4 & 186590896 & PZE-104110312 & $A, G$ & $1.74 \mathrm{E}-07$ & 0.004 & 0.18 & 0.03 & 4'phosphopante theinyl transferase \\
\hline 5 & GRMZM2G005308 & 6 & 160053330 & PZE-106113397 & $A, G$ & $1.96 \mathrm{E}-05$ & 0.074 & 0.24 & 0.02 & U3 small nucleolar ribonucleoprotein \\
\hline 6 & GRMZM2G151651 & 7 & 33447828 & SYNGENTA5726 & $\mathrm{G}, \mathrm{A}$ & $4.58 \mathrm{E}-08$ & 0.002 & 0.08 & 0.03 & \\
\hline 7 & GRMZM2G100107 & 7 & 91683817 & SYN16533 & $\mathrm{G}, \mathrm{A}$ & 2.07E-05 & 0.074 & 0.44 & 0.02 & SANT associated \\
\hline 8 & GRMZM2G100107 & 7 & 91684720 & PZE-107044973 & $A, G$ & $1.74 \mathrm{E}-05$ & 0.074 & 0.43 & 0.02 & SANT associated \\
\hline 9 & Intergenic & 7 & 91686972 & PZE-107044977 & $C, A$ & $1.43 \mathrm{E}-05$ & 0.074 & 0.44 & 0.02 & \\
\hline 10 & Intergenic & 7 & 92335869 & PZE-107045210 & $G, A$ & $3.64 \mathrm{E}-06$ & 0.034 & 0.21 & 0.02 & \\
\hline 11 & Intergenic & 8 & 37657703 & PZE-108032335 & $G, A$ & $1.43 \mathrm{E}-05$ & 0.074 & 0.36 & 0.02 & \\
\hline 12 & GRMZM2G158141 & 10 & 91956279 & PZE-110049068 & $\mathrm{G}, \mathrm{A}$ & $1.28 \mathrm{E}-05$ & 0.074 & 0.09 & 0.02 & Antifreeze protein \\
\hline
\end{tabular}

*False discovery rate-corrected $\mathrm{p}$-values

${ }^{\mathrm{a}}$ Minor allele frequency

annotated as the SANT domain-associated protein, which played an important role in disease resistance $[12,13]$. GRMZM2G158141 encoded antifreeze protein and may play direct role in plant defense [14]. GRMZM2G020254 encoded DNA-binding WRKY, which can cis regulate defense genes by signal transduction under biotic stress conditions [15].

\section{Haplotype-based association studies}

Gene-based haplotypes were constructed within the 7,551 genes which had at least 2 SNPs. On average a set of 4.9 haplotypes was defined in each of the 7,551 genes in present study. The haplotype analysis using these loci and phenotypic data from three disease parameters (i.e., AUDPC, mean rating and high rating) identified ten loci associated with resistance to NCLB. Of these loci, seven, five and seven were significantly associated with AUDPC, mean rating and high rating $\left(-\log _{10} P>3.88\right.$, $P=1 / 7,551$ loci), respectively (Fig. 3 ). Among the significant loci, four possible candidate genes (GRMZM2G089484, GRMZM2G020254, GRMZM2G097141 and GRMZM2G10 0107) were significantly associated with all three disease

Table 3 Candidate genes, chromosomal position and SNPs significantly associated with mean rating detected by SNP-based GWAS

\begin{tabular}{|c|c|c|c|c|c|c|c|c|c|c|}
\hline No. & Candidate gene & Chromosome & $\begin{array}{l}\text { Physical position } \\
\text { (AGP v.2) }\end{array}$ & SNP & Allele & $P$ value & $\mathrm{FDR}^{*}$ & $\mathrm{MAF}^{\mathrm{a}}$ & $\mathrm{R}^{2}$ & Annotation \\
\hline 1 & Intergenic & 1 & 264172677 & PZE-101213762 & $C, A$ & $6.44 \mathrm{E}-06$ & 0.029 & 0.18 & 0.02 & \\
\hline 2 & GRMZM2G150496 & 2 & 3735379 & PZE-102007366 & $\mathrm{G}, \mathrm{A}$ & 1.56E-05 & 0.048 & 0.18 & 0.02 & Inositol-pentakis-phosphate 2-kinase \\
\hline 3 & Intergenic & 3 & 103166745 & PZE-103062307 & $A, G$ & 3.05E-06 & 0.025 & 0.09 & 0.03 & \\
\hline 4 & GRMZM2G171605 & 4 & 186590896 & PZE-104110312 & $A, G$ & 5.33E-08 & 0.002 & 0.18 & 0.03 & 4'phosphopantetheinyl transferase \\
\hline 5 & AC233870.1_FG006 & 6 & 167018912 & PHM5529.7 & $C, A$ & $1.01 \mathrm{E}-05$ & 0.036 & 0.07 & 0.02 & \\
\hline 6 & GRMZM2G151651 & 7 & 33447828 & SYNGENTA5726 & $\mathrm{G}, \mathrm{A}$ & 1.06E-07 & 0.002 & 0.08 & 0.03 & \\
\hline 7 & GRMZM2G100107 & 7 & 91683817 & SYN16533 & $\mathrm{G}, \mathrm{A}$ & 5.79E-06 & 0.029 & 0.44 & 0.02 & SANT associated \\
\hline 8 & GRMZM2G100107 & 7 & 91684720 & PZE-107044973 & $\mathrm{G}, \mathrm{A}$ & 4.71E-06 & 0.027 & 0.43 & 0.02 & SANT associated \\
\hline 9 & GRMZM2G100107 & 7 & 91685110 & SYN16536 & $\mathrm{G}, \mathrm{A}$ & $6.81 \mathrm{E}-06$ & 0.029 & 0.43 & 0.02 & SANT associated \\
\hline 10 & Intergenic & 7 & 91686972 & PZE-107044977 & $A, C$ & 4.34E-06 & 0.027 & 0.44 & 0.03 & \\
\hline 11 & Intergenic & 7 & 92335869 & PZE-107045210 & $A, G$ & $1.38 \mathrm{E}-06$ & 0.021 & 0.21 & 0.03 & \\
\hline 12 & Intergenic & 9 & 25257190 & SYN28207 & $A, G$ & $3.18 \mathrm{E}-06$ & 0.025 & 0.1 & 0.03 & \\
\hline 13 & GRMZM2G020254 & 10 & 65416520 & PZE-110034333 & $A, G$ & 8.72E-06 & 0.034 & 0.21 & 0.02 & DNA-binding WRKY \\
\hline 14 & GRMZM2G158141 & 10 & 91956279 & PZE-1 10049068 & $\mathrm{G}, \mathrm{A}$ & 1.45E-05 & 0.048 & 0.1 & 0.02 & Antifreeze protein \\
\hline
\end{tabular}


Table 4 Candidate genes, chromosomal position and SNP significantly associated with high rating detected by SNP-based GWAS

\begin{tabular}{|c|c|c|c|c|c|c|c|c|c|c|}
\hline No. & Candidate gene & Chromosome & $\begin{array}{l}\text { Physical position } \\
\text { (AGP v.2) }\end{array}$ & SNP & Allele & $P$ value & $\mathrm{FDR}^{*}$ & $\mathrm{MAF}^{\mathrm{a}}$ & $R^{2}$ & Annotation \\
\hline 1 & GRMZM2G009715 & 3 & 87786034 & SYN15223 & $\mathrm{G}, \mathrm{A}$ & $1.63 \mathrm{E}-05$ & 0.040 & 0.14 & 0.02 & Potassium uptake protein TrkA \\
\hline 2 & Intergenic & 3 & 91910150 & PZE-103066271 & $A, G$ & $2.12 \mathrm{E}-05$ & 0.051 & 0.05 & 0.02 & \\
\hline 3 & Intergenic & 3 & 92149095 & PZE-103066064 & $C, A$ & 7.73E-06 & 0.039 & 0.07 & 0.02 & \\
\hline 4 & Intergenic & 3 & 103166745 & PZE-103062307 & $A, G$ & $1.14 \mathrm{E}-06$ & 0.021 & 0.09 & 0.03 & \\
\hline 5 & Intergenic & 3 & 103544700 & PZE-103062210 & $A, G$ & 9.29E-06 & 0.039 & 0.17 & 0.02 & \\
\hline 6 & Intergenic & 3 & 103769943 & PZE-103062159 & $A, C$ & 9.79E-06 & 0.039 & 0.13 & 0.02 & \\
\hline 7 & Intergenic & 3 & 146026075 & PZE-103087994 & $A, C$ & 1.35E-05 & 0.040 & 0.3 & 0.02 & \\
\hline 8 & Intergenic & 4 & 153495851 & PZE-104079154 & $\mathrm{G}, \mathrm{A}$ & 4.17E-06 & 0.039 & 0.25 & 0.03 & \\
\hline 9 & GRMZM2G080842 & 4 & 153499805 & SYN13972 & $A, G$ & 8.29E-06 & 0.039 & 0.24 & 0.02 & Mitochondrial carrier protein \\
\hline 10 & GRMZM2G080842 & 4 & 153500453 & SYN13976 & $C, A$ & 1.09E-05 & 0.039 & 0.24 & 0.02 & Mitochondrial carrier protein \\
\hline 11 & GRMZM2G080842 & 4 & 153500492 & SYN13977 & $A, G$ & $1.23 \mathrm{E}-05$ & 0.040 & 0.23 & 0.02 & Mitochondrial carrier protein \\
\hline 12 & GRMZM2G080842 & 4 & 153501980 & PZE-104079162 & $C, A$ & $1.04 \mathrm{E}-05$ & 0.039 & 0.25 & 0.02 & Mitochondrial carrier protein \\
\hline 13 & GRMZM2G080842 & 4 & 153502008 & PZE-104079163 & $A, G$ & 1.07E-05 & 0.039 & 0.24 & 0.02 & Mitochondrial carrier protein \\
\hline 14 & GRMZM2G171605 & 4 & 186590896 & PZE-104110312 & $A, G$ & $1.84 \mathrm{E}-06$ & 0.021 & 0.18 & 0.03 & 4'phosphopantetheinyltransferase \\
\hline 15 & GRMZM2G168807 & 5 & 165320067 & SYN16674 & $A, C$ & $1.50 \mathrm{E}-05$ & 0.040 & 0.32 & 0.02 & WW/Rsp5/WWP \\
\hline 16 & Intergenic & 5 & 187471551 & PZE-105130754 & $A, G$ & 1.47E-05 & 0.040 & 0.28 & 0.03 & \\
\hline 17 & GRMZM2G151651 & 7 & 33447828 & SYNGENTA5726 & $\mathrm{G}, \mathrm{A}$ & 1.79E-06 & 0.021 & 0.08 & 0.03 & \\
\hline 18 & GRMZM2G020254 & 10 & 65416520 & PZE-110034333 & $A, G$ & $1.24 \mathrm{E}-06$ & 0.021 & 0.21 & 0.03 & DNA-binding WRKY \\
\hline 19 & GRMZM2G089484 & 10 & 88686456 & PZE-110047506 & $\mathrm{G}, \mathrm{A}$ & $1.59 \mathrm{E}-05$ & 0.040 & 0.4 & 0.02 & Tyrosine protein kinase \\
\hline
\end{tabular}

*False discovery rate-corrected $\mathrm{p}$-values

${ }^{a}$ Minor allele frequency

parameters (Table 5), and three of them were annotated as resistance-related proteins (tyrosine protein kinase, DNAbinding WRKY and SANT domain-associated). When comparing the loci identified by single-SNP and haplotype-based associations, identical loci were also detected. For example, two candidate genes (GRMZM2G100107 and GRMZM2G0 20254) were significantly associated with at least two disease parameters based on both haplotype-based and SNP-based association analyses.

\section{Anderson-Darling (A-D) test for genome scanning}

The SNP data were further used for genome-wide scanning via A-D test to reveal the sources of resistance to NCLB. The total population was divided into three subgroups as described in the Methods section. Trait-marker association was performed by A-D test for each subgroup. As shown in the QQ and Manhattan plots (Additional file 5: Figure S2; Additional file 6: Figure S3; Additional file 7: Figure S4; Additional file 8: Figure S5), we found notable positive associations in subgroup 1 , in which $>100$ significant markers associated with different disease parameters were observed. In contrast, few significant associations were revealed in subgroup 2 and only small number of significant associations was observed in subgroup 3 . The predicted genes located within associated SNPs were identified using the MaizeGDB genome browser [16] or the http://ensembl.gramene.org/Zea_mays/Info/Index browser [17]. Here we listed 81 genes which were associated with at least two or three of the disease parameters (Additional file 9: Table S4). Among the predicted genes, 12 were related to plant defense (Table 6), which included antifreeze protein, PR transcriptional factor and a receptor-like kinase similar to those involved in basal defenses, and could be evaluated as potential candidate resistance genes. More importantly, when compared the defense genes with those identified by other two methods in present study (singlemarker and haplotype-based associations), we found GRMZM2G100107 was identical for all three analyses, and GRMZM2G171605 was identical for A-D test and single-marker based associations.

\section{Discussion}

Resistance to NCLB is a complex trait, and we know comparatively little about the genetic architecture in maize [18]. In the present study, a large number of lines were used to dissect the genetic architecture of resistance to NCLB. The germplasm covered a considerable amount of the genetic diversity found globally in maize, including 999 inbred lines from different sources, which were, most importantly, from multiple locations, allowing us to depict a clear global image. 

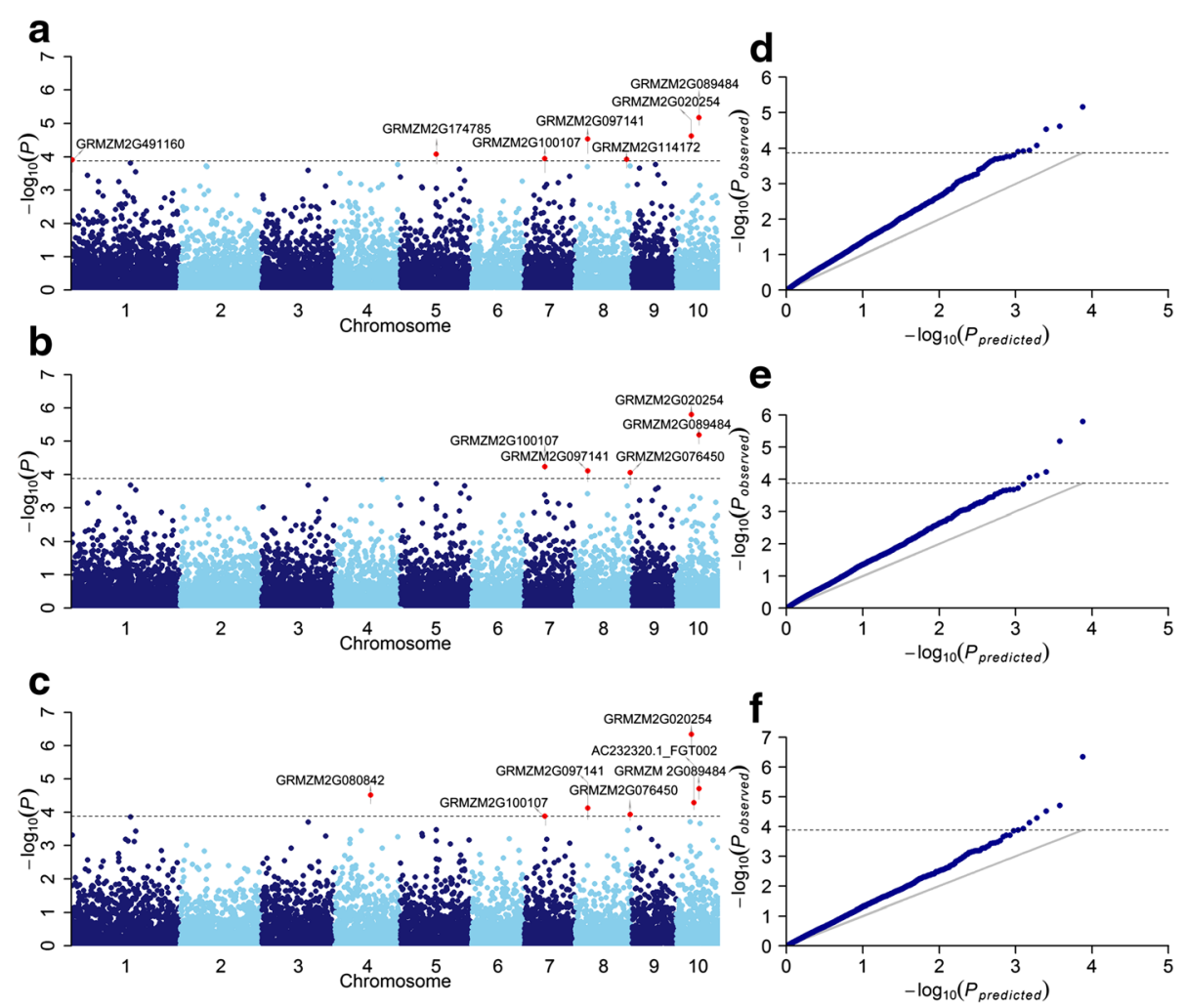

Fig. 3 Manhattan plots and QQ plots resulting from the haplotype-based GWAS for AUDPC, Mean Rating and High Rating. Manhattan plots for area under disease progress curve (AUDPC), Mean Rating and High Rating are shown in $\mathbf{a}, \mathbf{b}$ and $\mathbf{c}$, respectively. QQ plots for area under disease progress curve (AUDPC), Mean Rating and High Rating are shown in $\mathbf{d}$, e and $\mathbf{f}$, respectively

The high heritabilities of traits associated with resistance to NCLB revealed the potential of this panel for precisely mapping NCLB resistance genes. However, the population structure of the association panel is an important factor for GWAS. To minimize spurious correlations and associations attributable to genetic non-independence or genome-wide linkage disequilibrium (LD), we unified significant population structure information (contained in matrix Q) and pairwise relative kinship relationships among lines (contained in matrix $\mathrm{K}$ ) into the statistical model [19]. These results can significantly control the false positives, but the $\mathrm{Q}+\mathrm{K}$ model was extremely strict, and it was hard to find significant loci when using the Bonferroni threshold as the cutoff (data not shown). Therefore, we used a PCA $+\mathrm{K}$ instead of $\mathrm{Q}+\mathrm{K}$ model and observed significant loci for this disease. We further confirmed our results through different analysis methods, including a haplotype-based GWAS and A-D

Table 5 Chromosome, gene name and annotation of the genes for high rating, mean rating and AUDPC detected by haplotype-based GWAS

\begin{tabular}{lllll}
\hline No. & Chromosome & Gene name & Traits & Annotation \\
\hline 1 & 1 & GRMZM2G491160 & AUDPC & Mitochondrial carrier protein \\
2 & 4 & GRMZM2G080842 & High Rating & ENTH/VHS \\
3 & 5 & GRMZM2G174785 & AUDPC & SANT associated \\
4 & 7 & GRMZM2G100107 & High Rating, Mean Rating, AUDPC & Ubiquitin \\
5 & 8 & GRMZM2G097141 & High Rating, Mean Rating, AUDPC & BTB/POZ-like \\
6 & 8 & GRMZM2G114172 & AUDPC & DNA-binding WRKY \\
7 & 8 & GRMZM2G076450 & High Rating, Mean Rating & Tyrosine protein kinase \\
8 & 10 & GRMZM2G020254 & High Rating, Mean Rating, AUDPC & High Rating \\
9 & 10 & AC232320.1_FGT002 & High Rating, Mean Rating, AUDPC &
\end{tabular}


Table 6 A subset of 81 SNP loci found to be associated with resistance to NCLB by Anderson-Darling test

\begin{tabular}{lllllll}
\hline No. & Chromosome & $\begin{array}{l}\text { Physical position } \\
\text { (AGP v.2) }\end{array}$ & Gene ID & Subpopulation & Traits & Predicted gene function \\
\hline 1 & 1 & 198469464 & GRMZM2G123094 & subpop-3 & AUDPC, Mean Rating & Antifreeze protein \\
2 & 1 & 202300043 & GRMZM2G315375 & subpop-1 & AUDPC, Mean Rating & ABC transporter \\
3 & 1 & 202549145 & GRMZM2G112377 & subpop-1 & AUDPC, Mean Rating & Antifreeze protein \\
4 & 2 & 149335132 & GRMZM2G124524 & subpop-1 & AUDPC, High Rating, Mean Rating & PR transcriptional factor \\
5 & 3 & 135911049 & GRMZM2G153087 & subpop-1 & AUDPC, High Rating, Mean Rating & FYVE/PHD \\
6 & 3 & 145476628 & GRMZM2G397948 & subpop-1 & AUDPC, High Rating, Mean Rating & BTB/POZ \\
7 & 4 & 40358905 & GRMZM2G059266 & subpop-1 & AUDPC, High Rating, Mean Rating & Protein kinase C \\
8 & 4 & 186590896 & GRMZM2G171605 & subpop-1 & AUDPC, High Rating, Mean Rating & 4'phosphopantetheinyl transferase \\
9 & 7 & 7462912 & GRMZM2G406859 & subpop-1 & AUDPC, High Rating, Mean Rating & Antifreeze protein \\
10 & 7 & 91684720 & GRMZM2G100107 & subpop-1 & AUDPC, High Rating, Mean Rating & SANT associated \\
11 & 10 & 10290662 & GRMZM2G093895 & subpop-1 & AUDPC, High Rating, Mean Rating & Transcription factor \\
12 & 10 & 116680462 & GRMZM2G175525 & subpop-3 & AUDPC, High Rating, Mean Rating & PR transcriptional factor \\
\hline
\end{tabular}

tests for genome scanning. We observed several genes using different statistical approaches and determined that some of the genes were commonly associated with all of the traits based on highly correlated phenotypic data. Furthermore, the genes detected in our investigation caused minor effects and controlled a small portion of phenotypic variation. Therefore, we concluded that resistance to NCLB is controlled by several genes or QTLs, each of which has a minor effect, and that no single major gene that controls NCLB resistance is present in this germplasm.

Several qualitative genes have been identified in tropical and temperate germplasm backgrounds that confer resistance to NCLB. Most of these $\mathrm{Ht}$ genes (for $\mathrm{Hel}$ minthosporium turcicum, the former name of E. turcicum) are dominant or partially dominant, including Ht1, Ht2, Ht3, Ht4, Htn1, Htm1 [20] and the more recently identified $H t P$, as well as $r t$ [21]. Most of the genes were not cloned but mapped on chromosomes: Ht1 and HtP were mapped on the long arm of chromosome 2 (bin 2.08) [22, 23], Ht2 and Htn1 were mapped on the bins 8.05 and $8.06[24,25]$ and $r t$ was mapped on chromosome 3L (bin 3.06) [23]. We compared the physical locations of the predicted genes in the present study with the mapped $H t$ genes, and we found that $H t P$ was closely linked with GRMZM2G139463 and $r t$ was closely linked with GRMZM2G072780. More studies were required to understand the associations between the identified candidates and underlying genes. No doubt, present data provides good information for final cloning and validating these genes. Recently, two major QTLs, one on chromosome $1\left(q N L B 1.06_{T \times 303}\right)[26,27]$ and the other on chromosome 8 (qNLB8.06 $\left.{ }_{D K 888}\right)$, which is closely linked and functionally related to $\mathrm{Ht2}$ [28], have been fine-mapped and their locations narrowed to $3.6 \mathrm{Mb}$ and $0.46 \mathrm{Mb}$, respectively. However, we did not identify predicted genes within these regions in our population. Since high heritability of resistance to NCLB was observed in the association panel comprising of large number of lines, the major reason may be the number of markers in the population was limited( 50k). It was estimated that several million markers are required for a whole genome wide association study in maize [29], which makes us have no enough power to detect all the underlying loci affecting target traits.

Compared with single-marker association, haplotypebased association is expected to improve the power of detection when the marker density is limited. In the present study, the efficiency of LD mapping was improved by using a haplotype-based analysis, which was constructed from multiple SNP markers within the same gene. As a result, we identified a total of ten loci at a genome-wide level for the three disease parameters. Haplotypes may have the potential to be in higher LD with the causative variants than individual SNPs, especially when using mediumdensity SNP panels. Indeed, compared with the high heritabilities of the three traits, it was unlikely that resistance to NCLB was determined by only a small number of genes. It is more likely that resistance to NCLB is a complex trait involving a large number of loci, of which the candidates identified in this study may have the largest effects. Given the expected $>50,000$ maize genes and the 5-10 feasible SNPs per gene for a given haplotype, more markers are needed for precise LD mapping to accelerate the discovery of NCLB resistance genes in maize.

As we mentioned earlier, association mapping is a powerful tool to detect loci involved in the inheritance of traits, but identifying loci responsible for more complex traits is difficult. Population structure can result in spurious associations that result from unlinked markers being associated with causative loci [30]. Such associations can occur when the disease frequency varies across subpopulations, thus increasing the probability 
that affected individuals will be sampled. Any marker alleles that are present at a high frequency in the overrepresented subpopulation will be associated with the phenotype [31]. Recently, the A-D test was applied as a useful complement to GWAS of complex quantitative traits [32]. In present study, large number of markers was identified as having strong associations with the phenotype in the largest subgroup (subgroup 1), whereas the other two subgroups with less lines revealed few or small number of significant SNPs. Predicted genes containing the significant SNPs were identified, and 81 genes, including 12 genes that related to plant defenses, were found to be associated with two or three of the disease parameters. The A-D test balances false positives and statistical power, and it can be used to analyze complex traits such as resistance to NCLB in maize.

\section{Conclusion}

An association panel including 999 diverse lines was evaluated for resistance to NCLB in multiple environments, and a large number of resistant lines were identified and can be used as reliable resistance resource in maize breeding program. GWAS reveals that NCLB resistance is a complex trait under the control of many minor genes with relatively small effects. Identical genes for resistance to NCLB were detected using single-marker and haplotype-based associations, as well as A-D test. Pyramiding these genes in the same background may result in stable resistance to NCLB.

\section{Methods}

\section{Germplasm and phenotyping}

The population used in this study represents the global collection of maize germplasm consisting of 999 inbred lines of a diverse nature. Three types of inbred lines, CMLs, CIMBLs (CIMMYT breeding lines) and the Drought Tolerant Maize for Africa (DTMA) lines, from the CIMMYT germplasm collection were used in this study (Additional file 1: Table S1). These lines were evaluated at 12 locations during two consecutive years under artificially created epiphytotics of Exserohilum turcicum (Additional file 2: Table S2). A randomized complete block design was used at all locations with a maximum of three replications per location. Each plot consisted of a single 2-m row with 10 plants. Inocula for field inoculations were produced with sterile sorghum grains. Briefly, a population of a pure Exserohilum turcicum strain was obtained from infected leaves collected from the preceding year following the procedure of Asea et al. [33]. Pure cultures were grown on PDA medium and used to inoculate sterile sorghum grains to produce large volumes of inoculum. Inoculated bottles containing sterile sorghum were cultured at room temperature for 2 weeks, and then colonized grains were harvested and kept in the dark at room temperature until use.
Experimental plots were inoculated at the 4- to 6-leaf stage by placing 20-30 grains of Exserohilum turcicumcolonized sorghum in the leaf whorl. Data on disease severity were recorded, as were the corresponding diseased leaf areas of each plant. Whole plots were visually rated three times during the growing season for the percent NCLB severity using the CIMMYT scale (1-5), where $1.0=$ complete resistance, no lesions; $1.5=$ very slight infection, one to a few scattered lesions on lower leaves, covering $0-5 \%$ of the leaf surface only; $2.0=$ weak-tomoderate infection on lower leaves with a few scattered lesions on lower leaves, covering 6-20\%; 3.0 = moderate infection, abundant lesions on lower leaves and a few on middle leaves, with $21-50 \%$ of the leaf surface showing NCLB symptoms; $4.0=$ abundant lesions on lower and middle leaves extending to upper leaves, covering 51-80\% of the leaf surface and 5.0 = abundant lesions on all leaves, plant may be prematurely killed, lesions covering $>80 \%$ of the leaf surface [34].

\section{Statistical analyses}

The phenotypic multi-environmental data were subjected to the following methods to analyze different parameters. To minimize the effect of environmental variation, best linear unbiased prediction (BLUP) of each line were used for all three traits. BLUP estimation was by the model: $y=$ $\mathrm{Xb}+\mathrm{Zu}+\mathrm{e}$, where $\mathrm{X}$ and $\mathrm{Z}$ are incidence matrices. In general, $b$ represents fixed effects, $u$ represents random effects and e represents residuals. It is assumed that expectation are $E(y)=X b, E(u)=0, E(e)=0$. Residuals are independently distributed with variance, so $\mathrm{V}(\mathrm{e})=\mathrm{R}$, $\mathrm{V}(\mathrm{u})=\mathrm{G}$ and $\mathrm{COV}(\mathrm{u}, \mathrm{e})=0 . \mathrm{R}$ and $\mathrm{G}$ are known positive definite matrices. Hence

$$
\begin{aligned}
& V\left[\begin{array}{l}
u \\
e
\end{array}\right]=\left[\begin{array}{ll}
G & 0 \\
0 & R
\end{array}\right] \\
& u_{i}=\frac{\sigma_{A}^{2}}{\sigma_{e}^{2}+\sigma_{A}^{2}}\left(Y_{i}-\mu\right)
\end{aligned}
$$

$\sigma_{A}^{2}$ is variance of additive effects, $\sigma_{e}^{2}$ is variance of random effects, $Y_{i}$ is phenotypic observation of the $i$ individual and $\mu$ is overall mean. $u_{i}$ is BLUP value [35]. Analysis of variance was performed using SAS (Release 9.1.3; SAS Institute, Cary, NC, USA). The heritability of distinct traits was calculated as the ratio of the total genotypic to total phenotypic variances [36]. The average scoring data were used to calculate the mean rating, and the individual average data of each score at 7-day intervals was converted to the percent leaf area for the computation of AUDPC based on the formula suggested by Ceballos et al. [37] using the midpoint rule. AUDPC $=\Sigma_{i}=1^{n-1}\left[\left(t_{i}+1-t_{i}\right)\left(y_{i}+y_{i+1}\right) / 2\right]$, where $t$ is the time in days of each reading, $y$ is the percentage 
of affected foliage at each reading and $\mathrm{n}$ is the number of readings.

\section{Genotyping}

Genomic DNA extraction was performed using a modified CTAB protocol [38]. At least five leaves from each line were pooled and used for DNA extraction. All 999 lines were genotyped using GoldenGate assays (Illumina, San Diego, CA, USA) that were comprised of 56,110 authenticated SNPs, which were derived from the B73 reference sequence, evenly distributed across the 10 maize chromosomes [39]. The SNP genotyping was performed on an Illumina Infinium SNP genotyping platform at Cornell University Life Sciences Core Laboratories Center using the protocol developed by the Illumina Company.

\section{Population structure}

Population structure was estimated using the Bayesian Markov Chain Monte Carlo (MCMC) implemented in STRUCTURE [40, 41]. Briefly, SNPs with minor allelic frequencies $\geq 0.3$ were used first to select major SNPs, and then 1,000 markers were randomly selected from the whole set based on the physical length of each chromosome. Hypotheses were tested for subpopulations number from $K=1$ to $K=10$. For each $K$ value, seven independent runs were performed under the admixture model and correlated allele frequencies, with burn in time and MCMC replication number both to 100,000 . The $K$ value was determined by $\operatorname{LnP}(\mathrm{D})$ and hoc statistic delta $K$ based on the rate of change of $\operatorname{LnP}(\mathrm{D})$ between successive $K$ value [42]. Based on the simulation summary, bar plots were constructed with the lower value of $\operatorname{var}[\operatorname{LnP}(\mathrm{D})]$, and the populations were divided into three subgroups based on the delta $K$ following Yang et al. [43]. PCA was generated by setting the Genome Association and Prediction Integrated Tool- $\mathrm{R}$ package [44] and the $\mathrm{K}$ matrix was calculated using SPAGeDi software [45].

\section{SNP-based genome-wide association mapping}

To use the best quality data for different analyses, we did not analyze data from several lines that had high levels of missing genotypic data. In total, 981 lines were used in the final analysis, and all of the lines had high-quality phenotypic and genotypic data. SNP-based genome-wide association mapping was determined by using TASSEL (Trait Analysis by Association, Evolution and Linkage) software [46]. Of the 56,110 SNPs genotyped, 46,451 SNPs with minor allelic frequencies $\geq 5 \%$ were used for the GWAS. The MLM (PCA + K) model, which incorporated a kinship matrix $(\mathrm{K})$ along with the covariate $\mathrm{PC}$ (the first 10 principal components), was performed using MLM (P3D, no compression) [19, 43]. P value of each SNP was calculated and significance was defined at a uniform threshold of $\mathrm{P} \leq 2.15 \times 10^{-5}(P=1 / \mathrm{n} ; n=$ total markers used, which is roughly a Bonferroni correction). SNP with the lowest $\mathrm{P}$ value was reported for each significant locus, and the predicted genes located within associated SNPs were identified using the MaizeGDB genome browser [16] or the www.maizesequence.org/genome browser [17].

\section{Haplotype-based association studies}

In this study, SNP genotypes within the genes were selected to construct gene-based haplotypes. Since the number of SNPs in each gene varied (i.e., from one to fifteen), the genes which had only one SNP were discarded, and thus 7551 genes, each had $\geq 2$ SNPs, were selected to construct the haplotypes. Briefly, the genome was divided into genebased windows to determine the haplotypes of the linked SNPs. Each gene-based window was defined by all of the SNPs within a specific gene. If the gene contained more than five SNPs, a random subset of five SNPs was selected for the window. For subsequent analyses, each haplotype window was defined as a locus. Thus, 7551 gene-based windows were defined. Since there are more than one haplotypes within each gene, haplotypes with frequencies $<5 \%$ were discarded, then a multi-allelic test was performed for each set of haplotypes at a locus to identify the association between genes and traits. Haplotype-based GWAS was performed by using TASSEL software, and MLM was selected by taking both population structure PC (the first 10 principal components) and kinship (K) into account to avoid spurious associations.

\section{Anderson darling test}

Anderson-Darling test is a nonparametric statistical method and a variation of the Kolmogorov-Smirnov test [47] that gives weight to the tails of the distribution. In present study, Anderson-Darling test was conducted in each of three subgroups of the association panel. Briefly, each subpopulation was subjected to the $\mathrm{k}$-sample A-D ( $\mathrm{k}=$ number of samples) test, which is a variation of the Kolmogorov-Smirnov test [47] for genome screening. The observed $P$ value was used to construct QQ and Manhattan plots with SAS. The full details of this test have been published recently to dissect the genetic architecture of maize for 17 traits [32], and the software of A-D test can be performed using an R script and downloaded from http://www.maizego.org.

\section{Additional files}

Additional file 1: Table S1. The list of the lines and their phenotypic evaluation to NCLB in the association panel. (ODS $58 \mathrm{~kb}$ )

Additional file 2: Table S2. The field design of the association mapping panel for evaluation of resistance to NCLB. (ODS $13 \mathrm{~kb}$ )

Additional file 3: Table S3. The proportion of variance explained by ten groups of principal component analyses in association panel. (ODS $12 \mathrm{~kb}$ ) Additional file 4: Figure S1. Analysis of the population structure of maize inbred lines. a) Estimated $L n P(D)$ and $\Delta k$ of STRUCTURE analysis; 
b, c and $\mathbf{d}$ show the genetic variance controlled by the 56110 SNP makers for AUDPC, Mean Rating and High Rating, respectively. (DOC $119 \mathrm{~kb}$ )

Additional file 5: Figure S2. Manhattan plot for AUDPC in sub-group 1, 2 and 3, based on Anderson-Darling test. (DOC $66 \mathrm{~kb}$ )

Additional file 6: Figure S3. Manhattan plot for Mean Rating in sub-group 1,2 and 3, based on Anderson-Darling test. (DOC $64 \mathrm{~kb}$ )

Additional file 7: Figure S4. Manhattan plot for High Rating in sub-group 1,2 and 3, based on Anderson-Darling test. (DOC $66 \mathrm{~kb}$ )

Additional file 8: Figure S5. QQ plot for all the traits using Anderson-Darling test. The QQ plot for sub-groups 1, 2 and, 3 were shown in blue, green and red colors, respectively; while black line is the expected line. (DOC $42 \mathrm{~kb}$ )

Additional file 9: Table S4. SNP loci found to be associated with resistance to NCLB by GWAS using Anderson-Darling test. For the three disease parameters (AUDPC, mean rating and high rating), significant SNPs associated to 2 or 3 disease parameters were listed. (ODS $22 \mathrm{~kb}$ )

\section{Abbreviations}

A-D test: Anderson-Darling test; AUDPC: Area under disease progress curve; CIMBL: CIMMYT maize breeding line; CML: CIMMYT maize line; DTMA: Drought Tolerant Maize for Africa; GWAS: Genome wide association studies; K: Kinship; LD: Linkage disequilibrium; MLM: Mixed linear model; NCLB: Northern corn leaf blight; PCA: Principal component analyses; Q: Structure; QQ: Quantile-quantile; QTL: Quantitative trait locus; SNP: Single-nucleotide polymorphism.

\section{Competing interests}

The authors declare that they have no competing interests.

\section{Authors' contributions}

GM prepared the materials; JY designed the experiments and generated the raw data from the chip analysis; GM, LN, CM and DM participated in determining the phenotypes at all of the locations; JD, FA, GC and NY performed the genotypic and phenotypic analyses; HL help for haplotype analysis; JD and FA wrote the manuscript. All authors read and approved the final manuscript.

\section{Acknowledgements}

This work was supported by the National Natural Science Foundation of China (31161140347) and by the Drought-Tolerant Maize for Africa project, funded by the Bill and Melinda Gates Foundation.

\section{Author details \\ 'National Key Laboratory of Crop Genetic Improvement, Huazhong Agricultural University, Wuhan 430070, China. ${ }^{2}$ Institute of Crop Science, Chinese Academy of Agricultural Sciences, Beijing 100081, China. ${ }^{3}$ Global Maize Program, International Maize and Wheat Improvement Center (CIMMYT), Apdo. Postal 6-641, 06600 Mexico, DF, Mexico.}

Received: 13 May 2015 Accepted: 13 August 2015

Published online: 20 August 2015

\section{References}

1. Yan JB, Warburton M, Crouch J. Association mapping for enhancing maize (Zea mays L.) genetic improvement. Crop Sci. 2011;51:433-49.

2. Risch NJ. Searching for genetic determinants in the new millennium. Nature. 2000;405:847-56.

3. Rafalski JA. Association genetics in crop improvement. Curr Opin Plant Biol. 2010;13:174-80.

4. Korte A, Farlow A. The advantages and limitations of trait analysis with GWAS: a review. Plant Methods. 2013;9:29-37.

5. Stranger BE, Stahl EA, Raj T. Progress and promise of genome-wide association studies for human complex trait genetics. Genetics. 2011;187:367-83.

6. Mackay TFC, Stone EA, Ayroles JF. The genetics of quantitative traits; challenges and prospects. Nat Rev Gen. 2009;10:565-77.

7. Chang HS, Fan KC. Comparative studies on some biology and pathology of corn and broom corn isolates of Exserohilum turcicum (Pass) Leonard \& Suggs. Bot Bull Acad Sinica. 1986;27:209-18.
8. Poland JA, Bradbury PJ, Buckler ES, Nelson RJ. Genome-wide nested association mapping of quantitative resistance to northern leaf blight in maize. Proc Natl Acad Sci U S A. 2011;108:6893-8.

9. Raymundo AD, Hooker AL. Measuring the relationship between northern corn leaf blight and yield losses. Plant Dis. 1981;65:325-7.

10. Salvi S, Tuberosa R. To clone or not to clone plant QTLs: present and future challenges. Trends Plants Sci. 2005;10:297-304.

11. Balasubramanian S, Schwartz C, Singh A, Warthmann N, Kim MC. QTL mapping in new Arabidopsis thaliana advanced intercross-recombinant inbred lines. PLoS One. 2009:4, e4318.

12. Boyer LA, Latek RR, Peterson CL. The SANT domain: a unique histone-tail-binding module. Nat Rev Mol Cell Biol. 2004;5:158-63.

13. Berr A, Ménard R, Heitz T, Shen WH. Chromatin modification and remodelling: a regulatory landscape for the control of Arabidopsis defence responses upon pathogen attack. Cell Microbiol. 2012;14:829-39.

14. Hon WC, Griffith M, Mlynarz A, Kwok YC, Yang DS. Antifreeze proteins in winter rye are similar to pathogenesis-related proteins. Plant Physiol. 1995;109:879-89.

15. Ülker B, Somssich IE. WRKY transcription factors: from DNA binding towards biological function. Curr Opin Plant Biol. 2004;7:491-8.

16. Andorf CM, Lawrence CJ, Harper LC, Schaeffer ML, Campbell DA, Sen TZ. The Locus Lookup tool at MaizeGDB: identification of genomic regions in maize by integrating sequence information with physical and genetic maps. Bioinformatics. 2010;26:434-6.

17. Schnable PS, Ware D, Fulton RS, Stein JC, Wei F, Pasternak S, et al. The B73 maize genome: complexity, diversity, and dynamics. Science. 2009;326:1112-5.

18. Ali F, Yan JB. The phenomenon of disease resistance in maize and the role of molecular breeding in defending against global threat. J Integrated Plant Biol. 2012;55:134-51.

19. Yu J, Pressoir G, Briggs W, Vroh BI, Yamasaki M, Doebley JF, et al. A unified mixed-model method for association mapping that accounts for multiple levels of relatedness. Nat Genet. 2006;38:203-8.

20. Welz HG, Geiger HH. Genes for resistance to northern corn leaf blight in diverse maize populations. Plant Breed. 2000;119:1-14.

21. Ogliari JB, Guimarães MA, Geraldi IO, Camargo LEA. New resistance genes in the Zea mays L.-Exserohilum tucicum pathosystem. Genet Mol Biol. 2005;28:435-9.

22. Bentolila S, Guitton C, Bouvet N, Sailland A, Nykaza S, Freyssinet G. Identification of an RFLP marker tightly linked to the $\mathrm{Ht}$ 1 gene in maize. Theor Appl Genet. 1991;82:393-8.

23. Ogliari JB, Guirnaraes MA, Aranha Carnargo LE. Chromosomal locations of the maize (Zeamays L.) HtP and rt genes that confer resistance to Exserohilum turcicum. Genet Mol Biol. 2007;30:630-4.

24. Zaitlin D, DeMars S, Gupta M. Linkage of a second gene for NCLB resistance to molecular markers in maize. Maize Genet Coop Newsl. 1992;66:69-70.

25. Simcox KD, Bennetzen JL. Mapping the HtN resistance gene to the long arm of chromosome 8. Maize Genet Coop Newsl. 1993;67:118-9.

26. Chung CL, Longfellow JM, Walsh EK, Kerdieh Z, Esbroeck GV, Balint-Kurti P, et al. Resistance loci affecting distinct stages of fungal pathogenesis: use of introgression lines for QTL mapping and characterization in the maize-Setosphaeria turcica pathosystem. BMC Plant Biology. 2010;10:103-27.

27. Jamann TM, Poland JA, Kolkman JM, Smith LG, Nelson RJ. Unraveling genomic complexity at a quantitative disease resistance locus in maize. Genetics. 2014;198:333-44.

28. Chung $\mathrm{CL}$, Jamann T, Longfellow J, Nelson R. Characterization and fine-mapping of a resistance locus for northern leaf blight in maize bin 8.06. Theor Appl Genet. 2010;121:205-27.

29. Myles S, Peiffer J, Brown PJ, Ersoz ES, Zhang Z, Costich DE, et al. Association mapping: critical considerations shift from genotyping to experimental design. The Plant Cell. 2009;21:2194-202.

30. Lander ES, Schork NJ. Genetic dissection of complex traits. Science. 1994;265:2037-48

31. Pritchard JK, Rosenberg NA. Use of unlinked genetic markers to detect population stratification in association studies. Am J of Hum Gen. 1999;65:220-8.

32. Yang N, Lu YL, Yang XH, Huang J, Zhou Y, Ali F, et al. Genome wide association studies using a new nonparametric model reveal the genetic architecture of 17 agronomic traits in an enlarged maize association panel. PLoS Genet. 2014;10, e1004573.

33. Asea G, Vivek BS, Bigirwa G, Lipps PE, Pratt RC. Validation of consensus quantitative trait loci associated with resistance to multiple foliar pathogens of maize. Phytopathology. 2009;99:540-7.

34. The CIMMYT Maize Program. Maize diseases: A guide for field identification. 4th Edition. Mexico, D.F. CIMMYT; 2004. 
35. White T, Hodge G. Best Linear Unbiased Prediction: Introduction. In: Predicting Breeding Values with Applications in Forest Tree Improvement, vol. 33. Netherlands: Springer; 1989. p. 276-98.

36. Knapp SJ, Stroup WW, Ross WM. Exact confidence intervals for heritability on a progeny mean basis. Crop Sci. 1985;25:192-4.

37. Ceballos H, Deutsch JA, Gutierrez H. Recurrent selection for resistance to Exserohilum turcicum in eight subtropical maize populations. Crop Sci. 1991;31:964-71.

38. Murray MG, Thompson WF. Rapid isolation of high molecular weight plant DNA. Nucleic Acids Res. 1980;8:4321-5.

39. Ganal MW, Durstewitz G, Polley A, Bérard A, Buckler ES, Charcosset A, et al. A large maize (Zea mays L.) SNP genotyping array: development and germplasm genotyping, and genetic mapping to compare with the B73 reference genome. PLoS One. 2011;6(12):e28334.

40. Pritchard JK, Stephens M, Donnelly P. Inference of population structure using multilocus genotype data. Genetics. 2000;155:945-59.

41. Falush D, Stephens M, Pritchard JK. Inference of population structure using multilocus genotype data: linked loci and correlated allele frequencies. Genetics. 2003;164:1567-87.

42. Excoffier L, Laval G, Schneider S. Arlequin ver. 3.0: anintegrated software package for population genetics data analysis. Evol Bioinform Online. 2005;1:47-50.

43. Yang XH, Yan JB, Shah T, Warburton ML, Li Q, Li L, et al. Genetic analysis and characterization of a new maize association mapping panel for quantitative trait loci dissection. Theor Appl Genet. 2010;121:417-31.

44. Lipka AE, Tian F, Wang Q, Peiffer J, Li M, Bradbury PJ, et al. GAPIT: genome association and prediction integrated tool. Bioinformatics. 2012;28:2397-9.

45. Hardy OJ, Vekemans X. Spagedi: a versatile computer program to analyse spatial genetic structure at the individual or population levels. Mol Ecol Notes. 2002;2:618-20.

46. Bradbury PJ, Zhang Z, Kroon DE, Casstevens TM, Ramdoss Y, Buckler ES. TASSEL: software for association mapping of complex traits in diverse samples. Bioinformatics. 2007;23:2633-5.

47. Scholz F, Stephens M. K-sample anderson-darling tests. J Am Stat Assoc 1987;82:918-24.

\section{Submit your next manuscript to BioMed Central and take full advantage of:}

- Convenient online submission

- Thorough peer review

- No space constraints or color figure charges

- Immediate publication on acceptance

- Inclusion in PubMed, CAS, Scopus and Google Scholar

- Research which is freely available for redistribution 